\title{
Utilização de medicamentos na Pediatria: a prática de automedicação em crianças por seus responsáveis
}

\author{
Medication use in Pediatrics: the practice of self-medication in children by their parents
}

Gabriela Colonetti Beckhauser ${ }^{1}$, Juliana Medeiros de Souza', Cleidson Valgas², Anna Paula Piovezan², Dayani Galato ${ }^{3}$

\section{RESUMO}

Objetivo: Conhecer a automedicação em crianças moradoras de uma cidade da região Sul do Brasil.

Métodos: Estudo transversal com amostra selecionada por sorteio, inicialmente identificando as equipes de Estratégia Saúde da Família e, posteriormente, as residências que seriam visitadas. Foi adotado, para a entrevista com o responsável pelas crianças, um questionário para avaliar o perfil da criança, da família e da prática da automedicação. Para apresentação dos dados, adotou-se a estatística descritiva e o teste do qui-quadrado $(p<0,05)$.

Resultados: Entrevistaram-se os responsáveis pelas crianças em 83 domicílios. Foram coletadas informações de 121 crianças (seis meses a 14 anos). Dos entrevistados, $75 \%$ afirmaram já ter praticado a automedicação, sendo as mães responsáveis por $95 \%$ desses casos. Quanto às situações que motivaram a automedicação, praticidade (88\%), febre $(58 \%)$ e dor (12\%) foram as mais relatadas. O paracetamol $(45 \%)$ e a dipirona (15\%) foram os fármacos mais utilizados. A análise estatística evidenciou associação entre a reutilização de antigas prescrições e a idade da criança inferior a sete anos, bem como entre a utilização de medicamentos sem prescrição de profissional habilitado e morar em domicílios com mais de quatro pessoas.

Conclusões: A automedicação é uma prática frequente na população investigada, sendo geralmente mais comum em crianças de até sete anos e realizada principalmente pelas mães; esse fato sugere a necessidade de promover educação em saúde que vise à promoção do uso racional de medicamentos.

Palavras chaves: automedicação; criança; uso de medicamentos.

\section{ABSTRACT}

Objective: To assess self medication practice among children from a city in the Southern region of Brazil.

Methods: This is a cross-sectional study with a random sample. Initially, Family Health Strategy teams and later the residences that would be visited were raffled. A questionnaire about children and family characteristics and self medication practices was administered to caretakers. Descriptive statistics and chi-square test $(p<0.05)$ were used for data presentation.

Results: Child caretakers were interviewed in 83 households. Data from 121 children (aged six months to 14 years) were collected. Among caretakers, 75\% said they had practiced self medication; mothers accounted for $95 \%$ of these cases. As for self medication situations, convenience $(88 \%)$, fever $(58 \%)$ and pain $(12 \%)$ were the most reported. Acetaminophen (45\%) and dipyrone (15\%) were the most used drugs. Statistical analysis showed an association between the reuse of old prescriptions and child's age less than seven years and between the use of drugs without prescription from a qualified professional and households with more than four people.
Instituição: Núcleo de Pesquisa em Atenção Farmacêutica e Estudos de Utilização de Medicamentos (Nafeum) do Curso de Farmácia da Universidade do Sul de Santa Catarina (Unisul), Florianópolis, SC, Brasil

${ }^{1}$ Graduada em Farmácia, Estudante do Curso de Análises Clínicas na Unisul, Florianópolis (SC), Brasil

${ }^{2}$ Mestre em Ciências Farmacêuticas pela Universidade Federal de Santa Catarina (UFSC); Coordenador do Curso de Farmácia da Unisul, Florianópolis, SC, Brasil

${ }^{3}$ Doutora em Farmacologia pela UFSC; Professora de Mestrado em Ciências da Saúde da Unisul, Florianópolis, SC, Brasil

${ }^{4}$ Doutora em Química pela UFSC; Professora de Mestrado em Ciências da Saúde e Coordenadora do Nafeum da Unisul, Florianópolis, SC, Brasil
Endereço para correspondência:

Dayani Galato

Avenida José Acácio Moreira, 787 - Dehon

CEP 88704-900 - Tubarão/SC

E-mail: dayani.galato@unisul.br

Fonte de financiamento: Programa Universitário de Iniciação Científica (PUIC) da Unisul

Conflito de interesse: nada a declarar

Recebido em:21/7/09

Aprovado em:11/12/09 
Conclusions: Self medication is a common practice in the surveyed population, especially among children up to seven years of age and conducted primarily by mothers, suggesting the need to develop health education aimed at promoting the rational use of medicines.

Key-words: self medication; child; drug utilization.

\section{Introdução}

Para a Organização Mundial da Saúde (OMS) $)^{(1)}$, a automedicação consiste na seleção e utilização de medicamentos isentos de prescrição (sem tarja) para tratar doenças autolimitadas ou seus sintomas, estando inclusa no processo de autocuidado. Este último, por sua vez, compõe-se ainda de fatores como higiene (pessoal e geral), nutrição (tipo e qualidade do alimento ingerido), estilo de vida (atividade física e lazer), ambiente (condições de vida e hábitos sociais) e situação socioeconômica (renda, crenças culturais) $)^{(1)}$. Assim, a automedicação consiste na iniciativa de um sujeito ou de seu responsável em usar um medicamento que poderá trazer benefícios no tratamento de doenças ou alívio imediato de seus sintomas ${ }^{(2)}$.

Dentre as formas pelas quais a automedicação pode ser praticada, citam-se a aquisição de medicamentos sem receita, o compartilhamento dos medicamentos com outros integrantes da família ou círculo social, a reutilização de sobras de medicamentos de tratamentos anteriores e a utilização de antigas prescrições. Outras maneiras de realizá-la ocorrem quando há o descumprimento da prescrição, prolongando ou interrompendo precocemente o tratamento indicado ${ }^{(3)}$. Destaca-se, ainda, que esta prática é influenciada por amigos, familiares e balconistas de farmácia ${ }^{(4)}$.

Alguns autores observam que a automedicação pode ser realizada de forma responsável e benéfica quando é selecionada uma alternativa terapêutica (isenta de prescrição) com o auxílio e orientação de um farmacêutico para um problema de saúde autolimitado. Desse modo, a automedicação é adequada para o problema de saúde do paciente, na dose, concentração e tempo de tratamento adequado ${ }^{(5,6)}$. Por outro lado, a adoção inadequada dessa prática pode ocasionar danos à saúde do paciente, como o aparecimento de efeitos indesejáveis, agravos e mascaramento de doenças, interações medicamentosas, erros nas doses e intoxicações ${ }^{(7)}$. Esse fato torna-se particularmente relevante quando os sujeitos são crianças.
Estudo realizado por Pereira $e t a l^{(8)}$ demonstrou que $57 \%$ das crianças ou adolescentes participantes da pesquisa haviam sido automedicados. Reforçando essas informações, Bricks ${ }^{(9)}$ identificou o uso abusivo de medicamentos em Pediatria, especialmente de antibióticos, analgésicos e antitérmicos, principalmente para atuação no sistema respiratório. Outros autores $^{(9,10)}$ ainda destacam que os medicamentos empregados em Pediatria muitas vezes não possuem ações comprovadas nesse público. Morales-Carpi $e t a^{(11)}$ reforçam que, na maioria das vezes, os responsáveis pelas crianças possuem informações insuficientes e incongruentes sobre os medicamentos, mas, mesmo assim, adotam a automedicação em seus filhos.

Diante da importância do tema, o objetivo desta pesquisa foi conhecer o perfil da prática de automedicação em crianças, por seus responsáveis, em uma cidade do Sul de Santa Catarina, identificando fatores relacionados às situações e aos motivos dessa prática.

\section{Método}

Realizou-se um estudo transversal, baseado na técnica de entrevista com os responsáveis pelas crianças com idade entre zero e 14 anos, em seus respectivos domicílios. Para calcular o número de domićlíos que deveriam ser visitados, foram considerados os dados do Censo de $2000^{(12)}$, que registrou, no município de Tubarão, Santa Catarina, a existência de 22.129 crianças nessa faixa etária. Com esse total e considerando-se o número mínimo de uma criança por domicílio, foi possível determinar uma amostra representativa da população estudada igual a 114 (nível de confiança de $95 \%$, erro amostral de 10\%). Tal valor foi adotado por entender-se que, caso esse número de domicílios fosse visitado e mais do que uma criança residisse no mesmo, as respostas obtidas nas entrevistas com seus responsáveis teriam sua representatividade aumentada, uma vez que um maior número de crianças estaria sujeito à prática da automedicação. Além disso, o valor contém um fator de correção de 1,2 , isto é, mais $20 \%$, adotado para corrigir possíveis perdas ou recusas.

Para a composição da amostra, inicialmente foram sorteadas cinco das 22 unidades de Estratégia Saúde da Família (ESF) existentes no município. Realizou-se então o contato com seus responsáveis para obter o número de domicílios cadastrados em sua área de cobertura nos quais residiam crianças nas condições determinadas pelo estudo. O número calculado para a amostra de 114 domicílios foi dividido proporcionalmente entre essas ESF por meio de 
sorteio, utilizando a tabela de números aleatórios (EpiInfo 6.0 $)^{(13)}$. Na fase das entrevistas para coleta de dados, as residências foram visitadas com a ajuda dos agentes de saúde das respectivas microrregiões. Anteriormente, realizou-se um estudo piloto para a validação do instrumento de avaliação e da forma de abordagem aplicado em domicílios que não haviam sido sorteados para a pesquisa e, assim, os dados não foram incluídos nos resultados do presente relato.

Da amostra calculada, subtraíram-se 17 domicílios, nos quais os moradores não foram encontrados em quatro visitas realizadas em diferentes dias e períodos; outros seis, nos quais houve recusa em participar da pesquisa; e oito nos quais os moradores haviam se mudado. Dessa forma, a amostra final do estudo totalizou 83 domicílios.

$\mathrm{O}$ instrumento de coleta de dados continha perguntas abertas e fechadas com a finalidade de identificar o perfil das crianças pelos quais os entrevistados eram responsáveis (idade, sexo), bem como dos integrantes da família (escolaridade dos pais, número de pessoas no domicílio e renda) e, principalmente, identificar a prática da automedicação das crianças adotadas pelos mesmos (situações, motivações, responsáveis e alternativas adotadas).

Os medicamentos levantados no estudo foram classificados segundo a Anatomical Therapeutic Chemical (ATC). Os dados foram inseridos no programa EpiData ${ }^{(14)} \mathrm{e}$ analisados no programa Epi-Info 6.0 ${ }^{(13)}$, realizando-se teste do qui-quadrado para análise da associação entre o perfil da automedicação (uso de medicamentos sem prescrição e reutilização de antigas prescrições) e o perfil da criança ou da família entrevistada, adotando-se a mediana para compor os grupos das variáveis numéricas. $\mathrm{O}$ nível de significância estatística pré-estabelecido foi de $5 \%(p<0,05)$.
Este trabalho foi aprovado pelo Comitê de Ética em Pesquisa da Universidade do Sul de Santa Catarina.

\section{Resultados}

Foram realizadas entrevistas em 83 domicílios que possuíam entre dois e sete moradores, totalizando 327 moradores

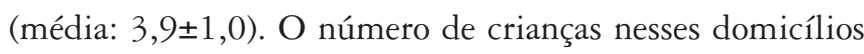
variou de um a três com média de $1,5 \pm 0,5$, somando, ao todo, 121 sujeitos com idades entre 0,5 a 14 anos $(7,3 \pm 4,1)$, sendo $52 \%$ de meninos.

O entrevistado, na maioria das vezes, foi representado pela mãe $(82 \%)$ ou avó (10\%); nos demais casos (8\%), foi representado pelo pai, padrasto ou irmão. A renda familiar referida pelos entrevistados variou de $\mathrm{R} \$ 50,00$ a $\mathrm{R} \$ 3.000,00$, tendo como média $\mathrm{R} \$ 1.291,00 \pm 710,00$. Em relação à escolaridade do pai, prevaleceu o médio completo (34\%) seguido do fundamental incompleto (30\%), sendo semelhante ao resultado encontrado em relação à escolaridade da mãe: ensino médio completo em $36 \%$ e fundamental incompleto em $35 \%$. As ocupações mais comuns do pai foram as assalariadas (70\%), seguida por profissões liberais (23\%), com resultados similares em relação à ocupação da mãe (classe assalariada: $46 \%$, do lar: 36\%).

Os dados relacionados ao perfil da prática da automedicação estão apresentados na Tabela 1. Quando investigadas as maneiras de alteração de prescrição médica (referida por $16,9 \%$ ), a forma mais comum foi a redução do tempo de tratamento $(50 \%)$, seguida pela diminuição da dose $(22 \%)$ ou pelo aumento da mesma (14\%), prolongamento do tempo de tratamento $(7 \%)$ e troca da ordem de horários na administração da medicação prescrita $(7 \%)$.

Tabela 1 - Formas de automedicação das crianças dos domicílios investigados

\begin{tabular}{lcc}
\hline & Descritor & $\mathbf{n ~ ( \% )}$ \\
\hline Alteração de prescrição médica $(n=83)$ & Sim & $14(16,9)$ \\
Reutiliza prescrições antigas ou sobras de medicamentos $(\mathrm{n}=83)$ & Não & $69(83,1)$ \\
& Sim & $53(63,9)$ \\
Utiliza medicamentos sem receita de um profissional prescritor $(\mathrm{n}=83)$ & Não & $30(36,1)$ \\
& Sim & $65(78,3)$ \\
A criança foi automedicada no último mês $(\mathrm{n}=83)$ & Não & $18(21,7)$ \\
& Sim & $30(36,1)$ \\
A criança já foi automedicada (vida toda) $(\mathrm{n}=80)$ & Não & $53(63,9)$ \\
& Sim & $62(77,5)$ \\
\hline
\end{tabular}


Os responsáveis pela prática da automedicação são as mães (95\%) e as avós (5\%). As situações, motivações, as influências e as estratégias utilizadas na automedicação (classes farmacológicas e medicamentos) estão descritas na Tabela 2.

Os dados relacionados às associações entre a automedicação (reutilização de antigas prescrições e utilização de medicamentos sem prescrição) e o perfil das crianças, dos responsáveis e do domicílio estão apresentados nas Tabelas 3 e 4 .

\section{Discussão}

A prevalência da automedicação no mês que antecedeu a entrevista foi de $36 \%$, semelhante àquela encontrada por Pereira $e t a^{(8)}$ no Estado de São Paulo, que observou 57\% de automedicação em um inquérito populacional com crianças e adolescentes. Da mesma forma, quando avaliado o principal responsável pela automedicação, também se identificou a mãe $e^{(8)}$; provavelmente tal situação ocorra pelo fato de a mãe ser a pessoa mais próxima da criança e a principal responsável pelo cuidado dos filhos.

A automedicação em crianças menores de sete anos merece destaque, pois foi observado que há maior chance de reutilizar antigas prescrições $(p=0,0013)$ em relação às crianças mais velhas, ou seja, a automedicação ocorre em maior prevalência nessa faixa etária. Tal fato pode ser consequência de um acompanhamento médico mais frequente, fazendo com que haja prescrições de medicamentos se necessário, bem como, quando há o reaparecimento de sintomas, a mãe utilize sobras de tratamento anteriores ou readquira os mesmos com prescrições já existentes.

Tabela 2 - Perfil da prática da automedicação realizada nas crianças

\begin{tabular}{llc}
\hline & Descritor & $\mathbf{n}(\%)$ \\
\hline Situações clínicas para a prática da & Febre & $53(57,6)$ \\
automedicação (n=92) & Dor & $25(27,2)$ \\
& Gripe & $4(4,3)$ \\
Fatores motivacionais (n=92) & Outras situações & $10(10,9)$ \\
& Falta de acesso ao Serviço de Saúde & $1(1,1)$ \\
Fatores que influenciam (n=92) & Considera como um problema de saúde simples & $10(10,9)$ \\
& Praticidade & $81(88,0)$ \\
& Motivação própria (“Já usei e não faz mal”) & $9(9,8)$ \\
Classes de medicamentos segundo ATC & Orientação do médico & $55(59,8)$ \\
(n=91) & Orientação na farmácia & $25(27,2)$ \\
& Influência de amigos, vizinhos e familiares & $3(3,3)$ \\
& B: trato alimentar e metabolismo & $1(1,1)$ \\
& G: sistema geniturinário e hormônios sexuais & $2(2,2)$ \\
& H: sistema hormonal & $1(1,1)$ \\
& J: anti-infecciosos de uso sistêmico & $2(2,2)$ \\
& M: sistema músculoesquelético & $5(5,5)$ \\
& N: sistema nervoso & $8(8,8)$ \\
& R: sistema respiratório & $66(75,5)$ \\
& Amoxicilina & $6(6,6)$ \\
& Ibuprofeno & $3(3,3)$ \\
& Ácido acetilsalicílico & $5(5,5)$ \\
& Dipirona & $3(3,3)$ \\
& Paracetamol & $14(15,4)$ \\
& Outros & $41(45,0)$ \\
& & $25(27,5)$ \\
\hline
\end{tabular}

ATC: Anatomical Therapeutic Chemical. 
Tabela 3 - Associação entre a reutilização de prescrições antigas (ReUPA) e o perfil das crianças, dos responsáveis e do domicílio

\begin{tabular}{|c|c|c|c|}
\hline & Descrição & n (\%) com ReUPA & Valor de $p$ \\
\hline \multirow[t]{2}{*}{ Sexo das crianças $(n=121)$} & Masculino & $42(67,7)$ & 0,698 \\
\hline & Feminino & $38(64,4)$ & \\
\hline \multirow[t]{2}{*}{ Idade das crianças $(n=121)$} & Até 7 anos & $50(79,4)$ & 0,001 \\
\hline & Mais de 7 anos & $30(51,7)$ & \\
\hline \multirow[t]{2}{*}{ Escolaridade do pai $(n=77)$} & Até fundamental completo & $26(68,4)$ & 0,277 \\
\hline & Médio ou superior & $22(56,4)$ & \\
\hline \multirow[t]{2}{*}{ Escolaridade da mãe $(n=83)$} & Até fundamental completo & $28(66,7)$ & 0,589 \\
\hline & Médio ou superior & $25(60,9)$ & \\
\hline \multirow[t]{2}{*}{ Renda da família ( $\mathrm{n=83)}$} & Até $\mathrm{R} \$ 1.000,00$ & $27(64,3)$ & 0,934 \\
\hline & Mais de $R \$ 1.000,00$ & $26(63,4)$ & \\
\hline \multirow[t]{2}{*}{$N^{\circ}$ de crianças no domicílio $(n=83)$} & Uma & $28(58,3)$ & 0,220 \\
\hline & 2 a 3 & $25(71,4)$ & \\
\hline \multirow[t]{2}{*}{$N^{\circ}$ de pessoas no domicílio $(n=83)$} & Até 4 pessoas & $40(66,7)$ & 0,389 \\
\hline & Mais de 4 pessoas & $13(56,5)$ & \\
\hline
\end{tabular}

Tabela 4 - Associação entre a utilização de medicamentos sem prescrição (UsemP) e o perfil das crianças, dos responsáveis e do domicílio

\begin{tabular}{|c|c|c|c|}
\hline & Descrição & n (\%) com UsemP & Valor de $p$ \\
\hline \multirow[t]{2}{*}{ Sexo das crianças $(n=121)$} & Masculino & $52(83,9)$ & 0,295 \\
\hline & Feminino & $45(76,2)$ & \\
\hline \multirow[t]{2}{*}{ Idade das crianças $(n=121)$} & Até 7 anos & $50(79,3)$ & 0,818 \\
\hline & Mais de 7 anos & $47(81,0)$ & \\
\hline \multirow[t]{2}{*}{ Escolaridade do pai $(n=77)$} & Até fundamental & $28(73,7)$ & 0,376 \\
\hline & Médio ou superior & $32(82,0)$ & \\
\hline \multirow[t]{2}{*}{ Escolaridade da mãe $(n=83)$} & Até fundamental completo & $32(76,2)$ & 0,635 \\
\hline & Médio ou superior & $33(80,4)$ & \\
\hline \multirow[t]{2}{*}{ Renda da família (n=83) } & Até $\mathrm{R} \$ 1.000,00$ & $33(78,5)$ & 0,954 \\
\hline & Mais de $\mathrm{R} \$ 1.000,00$ & $32(78,0)$ & \\
\hline \multirow[t]{2}{*}{$\mathrm{N}^{\circ}$ de crianças no domicílio $(\mathrm{n}=83)$} & Uma & $36(75,0)$ & 0,391 \\
\hline & 2 a 3 & $29(82,4)$ & \\
\hline \multirow[t]{2}{*}{$\mathrm{N}^{\circ}$ de pessoas no domicílio $(\mathrm{n}=83)$} & Até 4 pessoas & $43(71,7)$ & 0,018 \\
\hline & Mais de 4 pessoas & $22(95,7)$ & \\
\hline
\end{tabular}

Dessa forma, os dados da presente pesquisa divergem daqueles descritos por Pereira et al ${ }^{(8)}$, que demonstraram uma prevalência de consumo maior em sujeitos com mais de sete anos. Cabe ressaltar que, dependendo do local onde os estudos sobre utilização de medicamentos com crianças são realizados, pode haver diferença na faixa etária de maior uso, conforme indicado por Clavenna e Bona$\mathrm{ti}^{(15)}$. Um fato pertinente que pode ter contribuído para a reutilização de medicamentos em menores de sete anos estaria relacionado à condição fisiológica, já que, nessa idade, as crianças estão mais propensas a desenvolverem pequenos problemas de saúde, em especial, transtornos respiratórios ${ }^{(16)}$.

Uma parcela dos entrevistados (17\%) afirmou que já alterou a prescrição médica, sendo que a redução do tempo de tratamento foi a mais relatada $(50 \%)$, seguida da diminuição 
da dose (22\%). Isso pode ocorrer em função dos cuidadores identificarem melhoras nos sintomas apresentados pelas crianças. No entanto, chama-se a atenção para essa prática, que pode causar futuros problemas, como a resistência bacteriana $^{(17)}$.

Segundo os entrevistados, a automedicação é influenciada principalmente por antigas orientações do médico $(60 \%)$ ou por informações recebidas em estabelecimentos farmacêuticos (27\%). É importante destacar que, nesses estabelecimentos, os farmacêuticos, após a análise das informações do paciente (incluindo seu perfil e sintomas referidos), devem realizar a intervenção mais adequada, incluindo a orientação para procurar outro profissional de saúde, a recomendação de uma terapia nãofarmacológica (medidas higiênico-dietéticas) ou farmacológica (medicamento isento de prescrição). No entanto, o profissional farmacêutico deve acompanhar o paciente para identificar a efetividade e a segurança do tratamento ${ }^{(18)}$. Destaque-se que, segundo Vosgerau et al ${ }^{(19)}$, o farmacêutico tem papel fundamental na promoção do uso racional de medicamentos e cabe a ele, portanto, orientar, capacitar e apoiar ações de educação permanente dos outros membros da equipe de saúde para que o consumo de medicamentos não-prescritos e prescritos seja adequado e consciente.

Por outro lado, a adoção de antigas orientações do prescritor também pode apresentar problemas, já que nem sempre sintomas semelhantes requerem a mesma alternativa medicamentosa $^{(16)}$.

$\mathrm{O}$ fator que mais motivou a automedicação foi a praticidade (88\%): é mais prático ir à farmácia adquirir o medicamento do que marcar uma consulta com o médico para a mesma finalidade. Resultados semelhantes foram observados por Leite $e t a^{(20)}$, os quais observaram que, mesmo que os responsáveis tenham acesso ao médico, optam por adotar a automedicação como primeira opção antes de procurar os serviços de saúde, sejam eles públicos ou privados. No entanto, esse tipo de conduta pode trazer consequências como alguns efeitos indesejáveis ou mascaramento de doenças evolutivas, representando, portanto, um problema a ser prevenido ${ }^{(3)}$. É importante salientar que em outros estratos da população, como entre os idosos, essa atitude também tem a praticidade como principal motivação ${ }^{(5)}$.

As principais classes envolvidas na automedicação foram medicamentos analgésicos e antitérmicos que atuam no sistema nervoso (75\%), representados pelo paracetamol (45\%) e pela dipirona (15\%), seguidos pelo ibuprofeno $(6 \%)$ e pelo ácido acetilsalicílico (3\%). Tais medicamentos são citados como os mais comuns em outros estudos ${ }^{(7,21,22)}$, o que tem relação direta com os problemas de saúde para os quais são utilizados - neste estudo, a febre e a dor. Confirmando tais dados, Sukiennik et al ${ }^{(23)}$ demonstraram ser o paracetamol o antitérmico mais utilizado para o manejo da febre, com prevalência de $71 \%$. Allotey et $a^{(10)}$, que investigaram o uso de medicamentos sem prescrição em crianças australianas, também verificaram que o paracetamol é a medicação mais utilizada pelos responsáveis quando da automedicação de seus filhos.

Quanto à febre, Wannmacher e Ferreira ${ }^{(24)}$ afirmam que esse transtorno é simplesmente uma manifestação de uma determinada doença, sendo a defesa do organismo a uma possível infecção. No entanto, Sukiennik et al ${ }^{(23)}$ relatam que a febre é um dos principais motivos que resulta na procura de atendimento médico de urgência, pois os responsáveis acreditam na fragilidade pelo fato de o doente ser uma criança; destacam ainda que, mesmo com orientação médica, a maioria das crianças faz uso de doses indevidas de antitérmicos. O que torna ainda mais preocupante essa situação é que, segundo Allotey et al ${ }^{(10)}$, esse problema de saúde é o que mais leva os pais a adotarem a automedicação em seus filhos.

A amoxicilina é um antibiótico de uso sistêmico que, neste estudo, foi empregado na forma de automedicação. Ressalta-se que o mesmo é tarjado e, por consequência, não deveria ser adotado nessa situação, pois o uso desnecessário de antibióticos, além de desencadear diversos efeitos adversos, pode possibilitar a seleção de bactérias resistentes ${ }^{(17)}$.

Neste estudo verificou-se que não houve associação entre consumo de medicamentos por automedicação e a escolaridade dos pais, o que também foi verificado por Carvalho et $a l^{(3)}$. No entanto, quando a automedicação ocorre em adultos, Vilarino et al ${ }^{(25)}$ identificaram associação entre automedicação e maior grau de instrução. Em relação ao sexo, vários trabalhos apontam que as mulheres adotam mais essa prática ${ }^{(7,25)}$, no entanto, na Pediatria, não foi observada tal diferença ${ }^{(8)}$. $\mathrm{O}$ encontro de associação entre um maior número de moradores no domicílio e a adoção da automedicação pode ser explicado pelo fato de que, em famílias mais numerosas exista maior segurança nessa prática, provavelmente porque outros indivíduos que a compõem tenham passado por situações de saúde semelhantes, motivando a seleção de uma alternativa terapêutica.

As principais limitações da pesquisa referem-se ao tamanho relativamente pequeno da amostra e, dessa forma, os resultados devem ser interpretados com cautela. Contudo, destaca-se, na conclusão deste estudo, que a 
automedicação em crianças é bastante relevante e geralmente ocorre sob a responsabilidade das mães, em situações de febre e dor, com analgésicos e antitérmicos, sendo realizada por praticidade e por meio de antigas prescrições. Cabe ressaltar que essa prática é significativamente maior em crianças menores de sete anos e em domicílios com mais de quatro moradores. Portanto, este trabalho fornece uma visão do perfil de automedicação em crianças nessa região do Sul do Brasil.

\section{Referências bibliográficas}

1. World Health Organization (WHO) [homepage on the Internet]. The role of the pharmacist in self-care and self-medication [cited 2009 Mai 29]. Hague: World Health Organization; 1998. Available from: http://apps.who.int/medicinedocs/ en/d/Jwhozip32e/

2. Paulo LG, Zanine AC. Automedicação no Brasil. Rev Assoc Med Bras 1988;34:69-75.

3. Carvalho DC, Trevisol FS, Menegali BT, Trevisol DJ. Drug utilization among children aged zero to six enrolled in day care centers of Tubarão, Santa Catarina, Brazil. Rev Paul Pediatr 2008;26:238-44.

4. Kovacs FT, Brito MF . Disease perception and self medication in patients with scabies. An Bras Dermatol 2006;81:335-40.

5. Cascaes EA, Falchetti ML, Galato D. Perfil da automedicação em idosos participantes de grupos da terceira idade de uma cidade do sul do Brasil. ACM Arq Catarin Med 2008;37:63-9.

6. Andrade MA, Silva MV, Freitas O. Assistência farmacêutica como estratégia para o uso racional de medicamentos em idosos. Semina Cienc Biol Saude 2004;25:55-63.

7. Arrais PS, Coelho HL, Batista MC, Carvalho ML, Righi RE, Arnau JM. Aspects of self-medication in Brazil. Rev Saude Publica 1997;31:71-7.

8. Pereira FV, Bucaretchi F, Stephan C, Cordeiro R. Self-medication in children and adolescents. J Pediatr (Rio J) 2007;83:453-58.

9. Briks LF. [Judicious use of medication in children]. J Pediatr (Rio J) 2003;79 (Suppl 1):S107-14.

10. Allotey P, Reidpath DD, Elisha D. "Social Medication"and the control of children: a qualitative study of over-the-counter medication among Australian children. Pediatrics 2004;114:e378-83.

11. Morale-Carpi C, Julve Chover N, Carpi Lobatón R, Estañ L, Rubio E, Lurbe $\mathrm{E}$ et al. Medicamentos utilizados en pediatria extrahospitalaria: disponemos de información suficiente? An Pediatr (Barc) 2008;68:439-46.

12. Brasil - IBGE [homepage on the Internet]. Censo 2000 com divisão territorial 2001 [cited 2008 Mar 26]. Available from: http://www.ibge.gov.br/cidadesat/ default.php

13. Centers for Disease Control and Prevention - CDC. Epi info ${ }^{\mathrm{tm}}$ versão 3.32. Atlanta: CDC; 2005.

\section{Agradecimentos}

Ao apoio recebido da Secretária de Saúde do Município de Tubarão. Ao coordenador das ESF por permitir que se realizasse a coleta de dados. Às enfermeiras responsáveis pelas ESF, agentes de saúde e às famílias que participaram da mesma, bem como às professoras Simony Davet Müller, Silvana Cristina Trauthman e Irene Clemes Kulkamp pelas contribuições realizadas neste trabalho.

14. Lauritsen J M (ed.). EpiData Data Entry. Data management and basic statistical analysis system. Odense Denmark: EpiData Association, 2000 [cited 2008 ago 1]. Available from: http://www.epidata.dk

15. Clavenna A, Bonati M. Drug prescription to outpatient children: a review of the literature. Eur J Clin Pharmacol 2009;65:749-55

16. Tourinho FS, Bucaretchi F, Stephan C, Cordeiro R. Home medicine chests and their relationship with self-medication in children and adolescents. J Pediatr (Rio J) 2008;84:416-22.

17. Bricks LF, Ferrer AP. Treatment of acute respiratory infections in children: is nasal hypertonic saline use appropriated? Pediatria (São Paulo) 2007;29: 70-4.

18. Galato D, Galafassi LM, Alano GM, Trauthman SC. Responsible selfmedication: a reflection on the process of attending pharmaceutical. Brazilian Journal of Pharmaceutical Sciences. In press 2009.

19. Vosgerau MZ, Soares DA, Souza RK. Automedicação entre adultos na área de abrangência de uma Unidade de Saúde da Família. Lat Am J Pharm 2008;27:831-8.

20. Leite SN, Cordeiro BC, Thiesen D, Bianchini JP. Utilização de medicamentos e outras terapias antes de consulta pediátrica por usuários de unidade pública de saúde em Itajaí-SC, Brasil. Acta Farm Bonaerense 2006;25:608-12.

21. Loyola Filho AI, Uchoa E, Guerra HL, Firmo JO, Lima-Costa MF. Prevalence and factors associated with self-medication: the Bambui health survey. Rev Saude Publica 2002;36:55-62.

22. Musial DC, Dutra JS, Becker TC. A automedicação entre os brasileiros. SaBios-Rev Saude e Biol 2007;2:5-8.

23. Sukiennik R, Halpern R, Manica JL, Plentz FD, Bergamin G, Loss L et al. Antitérmicos na emergência pediátrica: estamos usando a dosagem adequada? Pediatria (São Paulo) 2006;28:175-83.

24. Wannmacher L, Ferreira MB [homepage on the Internet]. Febres: mitos que determinam condutas [cited 2009 Jun 30]. OPAS 2004;1:1-9. Available from: http://www.opas.org.br/medicamentos/site/UploadArq/HSE_URM_ FEB_0804.pdf

25. Vilarino JF, Soares IC, Silveira CM, Rödel AP, Bortoli R, Lemos RR. [Selfmedication profile in a city in South Brazil]. Rev Saude Publica 1998;32:43-9. 